\title{
SEROPREVALENCIA DE LEPTOSPIROSIS Y CARACTERÍSTICAS ASOCIADAS EN AGRICULTORES DE ARROZ DE UNA REGIÓN TROPICAL DEL PERÚ
}

\author{
Jorge O. Alarcón-Villaverde ${ }^{1, a, b}$, Franco Romani-Romani ${ }^{1, a}$, Romina A. Tejada ${ }^{1, a, c}$, Paolo Wong-Chero ${ }^{1, a}$, \\ Manuel Céspedes-Zambrano 2,d,e
}

\begin{abstract}
RESUMEN
Objetivos. Estimar la seroprevalencia de leptospirosis en agricultores dedicados al cultivo de arroz del valle del Alto Mayo, región San Martín e identificar anticuerpos para los serovares circulantes y los factores asociados a la infección. Materiales y métodos. Se realizó un estudio transversal entre octubre y noviembre de 2010 en una muestra de 254 agricultores procedentes de las once comisiones de regantes del valle de Alto Mayo. Se recolectó una muestra de sangre y se aplicó un cuestionario que incluyó variables sociodemográficas, y características del hogar y del trabajo. Se determinó la presencia de anticuerpos contra leptospira usando la prueba de microaglutinación y ELISA IgM. Resultados. La seroprevalencia determinada por microaglutinación fue del 64,6\% (IC 95\%: 58,6-70,6). La prueba ELISA IgM fue reactiva en 15,0\% (IC 95\%: 10,5-19,5). Los tipos de serovar más frecuentes fueron Icterohaemorrhagiae, Autumnalis, Australis, Panama y Grippotyphosa. Los factores asociados independientemente fueron la manipulación de roedores (OR ajustado [ORa] de 7,9, IC 95\%: 1,6-37,9), trabajar descalzo (ORa de 2,9, IC 95\%: 1,2-6,8) y el sexo masculino (ORa de 4,5, IC 95\%: 1,3-15,3). Conclusiones. El valle del Alto Mayo es un área hiperendémica de leptospirosis. Los factores asociados más importantes fueron la práctica de trabajar descalzo y la de manipular roedores en los campos de cultivo. En consecuencia, se recomienda implementar intervenciones que incidan en estos dos factores a fin de reducir el riesgo de infección en los cultivadores de arroz.
\end{abstract}

Palabras clave: Leptospirosis; Prevalencia; Enfermedades de los trabajadores agrícolas; Perú (fuente: BIREME).

\section{LEPTOSPIROSIS SEROPREVALENCE AND ASSOCIATED FEATURES IN RICE FARMERS OF TROPICAL REGION OF PERU}

\begin{abstract}
Objectives. To estimate the seroprevalence of leptospirosis in rice farmers of the Alto Mayo Valley in the Peruvian region of San Martín and to identify factors associated with infection. Materials and methods. A cross-sectional study was conducted between October and November of 2010 on a sample of 254 farmers from eleven irrigation committees in the Alto Mayo Valley. A blood sample was collected as well as sociodemographic, household and labor characteristics in the form of a questionnaire. The presence of antibodies against leptospira was determined using the microscopic agglutination test and the IgM ELISA. Results. The seroprevalence by agglutination was $64.6 \%$ (95\% Cl: 58.6-70.6). The IgM ELISA test was reactive in 15,0\% (95\% Cl: 10.5-19.5). The most frequent serovar were Icterohaemorrhagiae, Autumnalis, Australis, Panama and Grippotyphosa. Independently associated factors were handling rodents (adjusted OR (aOR): 7.9 ; 95\% Cl: 1.6-37.9), working barefoot (aOR: 2.9; 95\% Cl: 1.2-6.8) and male sex (aOR: 4.5; 95\% Cl: 1.3-15.3). Conclusions. The Alto Mayo Valley is a hyperendemic for leptospirosis. The most important factors were associated with working barefoot and handling rodents in crop fields. Consequently, it is recommended to implement interventions that affect these two factors in order to reduce the risk of infection in rice farmers.
\end{abstract}

Key words: Leptospirosis; Prevalence; Agricultural workers' diseases; Peru (source: MeSH NLM).

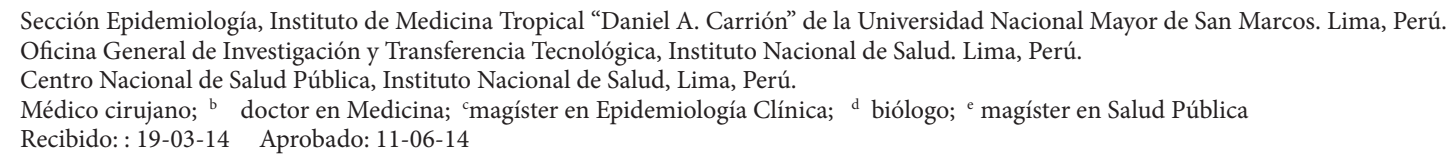

Citar como: Alarcón-Villaverde JO, Romani-Romani F, Tejada RA, Wong-Chero P, Céspedes-Zambrano M. Seroprevalencia de leptospirosis y características asociadas en agricultores de arroz de una región tropical del Perú. Rev Peru Med Exp Salud Publica. 2014;31(2):195-203. 


\section{INTRODUCCIÓN}

La leptospirosis es una zoonosis de distribución mundial ${ }^{(1,2)}$, causada por especies patógenas de Leptospira ${ }^{(3)}$. Las regiones tropicales y subtropicales son las mayormente afectadas, tanto en zonas urbanas como rurales ${ }^{(2,4-7)}$. Existen condiciones que favorecen la transmisión como el clima, la presencia y contacto con animales reservorios, las prácticas de agricultura, las condiciones sanitarias del ambiente y las prácticas de higiene en el hogar ${ }^{(5,8-11)}$. La leptospirosis se presenta con frecuencia en personas dedicadas a la minería, trabajadores de desagüe, ganaderos, carniceros, veterinarios y agricultores. En estos casos, es considerada una enfermedad ocupacional, donde el factor común es la exposición directa o indirecta a tejido animal u orina contaminada con la bacteria ${ }^{(1,2)}$.

El curso clínico de la leptospirosis depende de varios factores que interactúan, entre ellos el serovar infectante, la dosis de leptospiras infectantes, la densidad de reservorios, el tipo y frecuencia de exposición, el estado nutricional del paciente y la disponibilidad de atención médica (12). En zonas endémicas se ha observado que una considerable proporción de individuos presentan formas subclínicas de leptospirosis y no son diagnosticados ${ }^{(12-15)}$. En este contexto, el valor de las pruebas diagnósticas es relativo al momento que se apliquen y de la interacción de los factores antes descritos. En los estudios de prevalencia se usa con frecuencia pruebas serológicas, como la prueba de microaglutinación (MAT), que detecta anticuerpos serovar específicos, o la detección de anticuerpos inmunoglubulina M (IgM) (16). La MAT tiene una sensibilidad del $90 \%$ y una especificidad mayor al $90 \%$; la positividad a esta prueba se presenta a partir del día 10 al 12 de la infección y puede persistir por años. Por ello, un solo resultado de MAT positivo no puede diferenciar casos de infección reciente de infecciones previas (1). El ELISA IgM tiene una sensibilidad mayor a $90 \%$ y una especificidad de 88 a $95 \%$, puede detectar anticuerpos antes que MAT (4 a 5 días) y persiste hasta los cinco meses ${ }^{(1)}$. Cuando se usa como prueba diagnóstica necesita confirmación por MAT ${ }^{(2,11,16)}$.

La incidencia de leptospirosis varia de 0,1 a 1 por 100000 habitantes en climas templados, mientras que en países tropicales puede ser mayor de 10, pudiendo llegar a valores mayores en situaciones de epidemia y en grupos de riesgo de alta exposición ${ }^{(2,9)}$. En el Perú, según el sistema nacional de vigilancia epidemiológica, la incidencia acumulada de leptospirosis ha ido en aumento: en 2011 la incidencia fue de 1,0 por 100 mil habitantes; en 2012, fue 6,3, y en 2013 (hasta la semana 50) 8,6 (17). Los casos fueron notificado por casi todas las regiones del Perú, principalmente de la selva alta y la Amazonía (Amazonas, Loreto, Madre de Dios, San Martín), con tasas de incidencia de más de 50 casos por 100 mil habitantes ${ }^{(18)}$.

Sin embargo, es muy probable que el número de casos de leptospirosis que ocurren en el país sea mayor, como lo sugieren los diversos estudios de seroprevalencia realizados tanto en pacientes febriles $(36,6 \%$ en Madre de Dios, provincia de Manu, año 2000 (19); 30,6\% en Ayacucho, provincia de La Mar, distrito de Ayna, año $2004{ }^{(20)}$, como en población general $(31,3 \%$ en Ucayali, provincia Coronel Portillo, año 2001 (21); 28\% en Loreto, distrito de Belén (12); y 25,2\% en cuatro provincias de San Martín, año $2000{ }^{(22)}$ ). En la mayoría de casos el método empleado fue MAT, excepto en lquitos y San Martín donde se usó el método de ELISA (IgG e IgM). En dos de estos estudios se encontró que el ser agricultor es un factor de riesgo para la leptospirosis ${ }^{(21,22)}$, pero no se identificaron otros factores de riesgo.

Los objetivos del presente estudio fueron estimar la seroprevalencia de leptospirosis en agricultores dedicados al cultivo de arroz del valle del Alto Mayo, región San Martín, donde este cultivo es intensivo, e identificar anticuerpos para los serovares circulantes y los factores asociados a la mayor prevalencia de infección.

\section{MATERIALES Y MÉTODOS}

Se realizó un estudio transversal en arroceros del valle del Alto Mayo, región San Martín, entre octubre y noviembre de 2010. El valle tiene una extensión de 24 666,68 hectáreas, que comprende las provincias de Rioja y Moyobamba. Se sitúa entre 848 y 1000 metros de altitud, su clima es subtropical y su temperatura media anual es $22,5^{\circ} \mathrm{C}\left(16,5-28,4{ }^{\circ} \mathrm{C}\right){ }^{(23)}$. Los trabajadores están organizados en una Junta de Usuarios conformada por 7610 socios distribuidos en once comisiones de regantes.

El estudio se hizo en una muestra de trabajadores mayores de 18 años, con más de un mes de residencia. Se usó la relación de afiliados a la Junta de Usuarios como marco muestral. El tamaño se estimó para una selección aleatoria estratificada con afijación de varianza mínima de Neyman. Los parámetros tomados en cuenta fueron: prevalencia esperada de $56 \%{ }^{(24)}$, margen de error del $5 \%$ y nivel de significancia de 0,05 . La muestra estimada fue de 254 agricultores, procedentes de las once comisiones de regantes. En los casos en que un participante 
seleccionado no pudo ser contactado, se invitó a otro trabajador de la misma comisión. Para la recolección de datos se usó un cuestionario estructurado, aplicado por médicos capacitados.

Para la determinación de la infección se obtuvo $7 \mathrm{~mL}$ de sangre venosa periférica por punción en la vena antecubital de cualquiera de los antebrazos; la colección fue realizada en un tubo sin anticoagulante en el local de cada comisión. Las muestras fueron inmediatamente transportadas al laboratorio del Hospital Regional I de Moyobamba del Ministerio de Salud para realizar la centrifugación y separación del suero en crioviales, los cuales fueron congeladas a $-20{ }^{\circ} \mathrm{C}$ hasta su transporte y análisis en el Laboratorio de Zoonosis Bacteriana del Centro Nacional de Salud Pública del Instituto Nacional de Salud (INS).

Las muestras fueron sometidas a dos métodos serológicos: ELISA IgM y MAT. Para ELISA IgM se utilizó el método de ensayo del INS, el cual utilizada el kit de ELISA IgM elaborado y estandarizado por el INS (Lima-Perú) ${ }^{(25,26)}$. La prueba MAT fue realizada utilizando serovares que pertenecen a los serogrupos: Andamana, Bratislava, Autumnalis, Ballum, Bataviae, Canicola, Celledoni, Pomona, Hebdomadis, Cynopteri, Djasiman, Georgia, Grippotyphosa, Icterohaemorrhagiae, Javanica, Pyrogenes, Sejroe, Tarassovi y un nuevo serovar (Varillal) ${ }^{(27)}$. Los títulos de MAT $\geq 1: 100$ fueron considerados seropositivos e indicadores de exposición actual o previa a leptospiras.

Se consideró como sintomático a los agricultores que al momento de la evaluación clínica presentaron, al menos, uno de los siguientes síntomas o signos sugestivos de leptospirosis, incluidos en los criterios de Faine modificados: dolor de cabeza de inicio súbito, fiebre, sufusión conjuntival bilateral, meningismo, dolor muscular en pantorrillas e ictericia ${ }^{(28)}$.

Los datos demográficos incluyeron: edad, sexo, lugar de nacimiento, lugar de procedencia, tiempo de residencia en la región, grado de instrucción, ocupación, e ingreso mensual personal. En el caso del hogar, se tomó en cuenta el nivel de protección de la vivienda para el ingreso de reservorios, categorizado como alto (techo, piso y pared de cemento y ladrillos), medio (al menos una de las estructuras de material diferente al cemento y ladriIlo), y bajo (ninguna de las estructuras de cemento o ladrillo); el saneamiento (eliminación de excretas y orina, cercanía a alcantarillas, desagües abiertos o basurales, presencia de reservorios animales); y hábitos relacionados con el almacenamiento de alimentos, el lugar donde se bañan y la crianza de animales.
En cuando al trabajo, se consideró el tiempo de trabajo como cultivador de arroz, las horas de trabajo por día, el uso de calzado, los antecedentes de heridas durante el trabajo y la manipulación de roedores en el campo de cultivo.

Se hizo el análisis descriptivo de las características clínicas y epidemiológicas. Se estimó la seroprevalencia de leptospirosis con ambos métodos de laboratorio. Posteriormente, se realizó el análisis bivariado. Para el estudio de asociaciones se usó la prueba de chi cuadrado y la razón de probabilidades (odds ratio) con un intervalo de confianza al $95 \%$ y nivel de significancia del 0,05. Se elaboró un modelo multivariado de regresión logística, al cual ingresaron las variables estadísticamente asociadas a la seroprevalencia en el análisis bivariado. Se incluyó en el modelo la variable sexo, luego de que el análisis estratificado y multivariado mostró su efecto modificador sobre la variable manipulación de roedores. Se emplearon los programas estadísticos Epi-Dat, Epi Info versión 7.1.3.0 y SPSS versión 15 para Windows (2006, U.S.A. SPSS Inc).

El estudio fue aprobado por el comité de ética del Instituto de Medicina Tropical "Daniel A. Carrión" (IMT-UNMSM) y del INS. Se trabajó en coordinación con la Dirección Regional de Salud, el Hospital Regional I de Moyobamba, el Proyecto Especial Alto Mayo (PEAM), la Junta de Usuarios de la cuenca del Alto Mayo, los presidentes de cada comisión y los líderes de la comunidad. Previa a la obtención del consentimiento informado escrito, se realizó una charla grupal en cada comisión donde se explicó los objetivos y procedimientos del estudio. Luego se procedió a invitar a los agricultores seleccionados en forma individual.

\section{RESULTADOS}

\section{CARACTERISTTICAS DEMOGRÁFICAS DE LA POBLACIÓN DE ESTUDIO}

Participaron en el estudio 260 agricultores de arroz. La muestra se caracterizó por ser predominantemente masculina (85\%), mayor de 39 años $(70 \%)$, con bajo nivel de escolaridad y bajos ingresos económicos. El $81 \%$ fueron migrantes de otras regiones, principalmente Cajamarca $(\mathrm{N}=136,52,5 \%)$, Amazonas $(\mathrm{N}=44,17,0 \%)$ y Lambayeque ( $N=15,5,8 \%)$. La mediana del tiempo de residencia en el área estudiada fue de 25 años ( $72 \%$ de entrevistados residían más de 19 años en la región) (Tabla 1). 
Tabla 1. Características demográficas de los agricultores de arroz del valle del Alto Mayo, 2010

\begin{tabular}{lcc}
\hline Variable & N & (\%) \\
\hline Sexo masculino & 221 & $(85,0)$ \\
$\begin{array}{l}\text { Rango de edad } \\
\quad \leq 39\end{array}$ & 78 & $(30,0)$ \\
$\quad 40-59$ & 126 & $(48,5)$ \\
$\quad \geq 60$ & 56 & $(21,5)$ \\
\hline Natural de San Martín & 48 & $(18,5)$ \\
Años de residencia en área estudiada & & \\
$\quad \leq 19$ & 73 & $(28,1)$ \\
$\quad 20-29$ & 80 & $(30,8)$ \\
$\quad \geq 30$ & 107 & $(41,2)$ \\
Grado de instrucción secundaria o superior & 100 & $(38,8)$ \\
Ingreso mensual personal mayor a S/. 500 & 83 & $(32,3)$ \\
\hline
\end{tabular}

\section{CONDICIONES DEL HOGAR Y TRABAJO}

Según la clasificación de la vivienda descrita, el 41,9\% (109) habitaba en viviendas poco protegidas; el $38,5 \%$ (100) en viviendas medianamente protegidas, y el $19,6 \%$ (51) en viviendas altamente protegidas. El 66,4\% (172/259) contaba con agua entubada de la red pública y solo el $26,9 \%$ (70/260) contaba con servicio de desagüe. El 36,3\% (94/259) tenía su vivienda cerca a los campos de cultivo; el 36,5\% (93/255) cerca de áreas inundables; el 13,1\% (34/260) cerca de basurales, y el $37,3 \%$ (97/260) cerca de alcantarillas o desagües abiertos. La mayoría de entrevistados refirió haber observado ratas dentro $(94,2 \%, 245 / 260)$ y alrededor del hogar $(84,6 \%, 220 / 260)$.

El 93,5\% (243/260) refirió cubrir los alimentos dentro del hogar y el 72,3\% (188/260) informó haber observado que sus alimentos fueron consumidos por roedores. El $26,5 \%(69 / 260)$ de agricultores almacena comida en el peridomicilio. El 74,6\% (188/252) refirió que se baña en casa; pero hay una proporción que lo hacía en el río o la quebrada $(9,2 \%, 22 / 240)$; en el canal de regadío $(16,2 \%, 38 / 235)$, o en pozo o manantial $(5,9 \%, 14 / 237)$. La mayoría cría perros $(73,8 \%, 192 / 260)$; una proporción menor cría ganado vacuno $(26,9 \%, 70 / 260)$ y cerdos $(20,4 \%, 53 / 260)$.

En la Tabla 2 se describen las condiciones de trabajo de esta población. El 73\% tenía más de 15 años dedicados al cultivo de arroz (media \pm DE: $24,2 \pm 13,16$ años). La jornada de trabajo es de seis a más horas. Una alta proporción refirió no usar calzado en el trabajo $(58,5 \%)$. El $90 \%$ de agricultores refirió haber sufrido heridas en el trabajo, siendo las más frecuentes: cortantes $(59,6 \%$, $137 / 230)$, punzocortantes $(21,3 \%, 49 / 230)$, punzantes
Tabla 2. Condiciones de trabajo de los agricultores de arroz del valle del Alto Mayo, 2010

\begin{tabular}{lcc}
\hline Variable & N & (\%) \\
\hline $\begin{array}{l}\text { Años como agricultor de arroz } \\
\quad<15\end{array}$ & 59 & $(22,9)$ \\
\hline $15-29$ & 102 & $(39,5)$ \\
$\quad \geq 30$ & 97 & $(37,6)$ \\
$\begin{array}{l}\text { Horas de trabajo por día } \\
\quad \leq 5 \mathrm{~h}\end{array}$ & 49 & $(19,0)$ \\
\hline $6-8 \mathrm{~h}$ & 172 & $(66,7)$ \\
$\quad>8 \mathrm{~h}$ & 37 & $(14,3)$ \\
$\begin{array}{l}\text { Uso de calzado } \\
\quad \text { Descalzo }\end{array}$ & 152 & $(58,5)$ \\
$\quad \begin{array}{l}\text { Usa botas } \\
\quad \text { Usa zapatos, zapatillas o sandalias }\end{array}$ & 73 & $(28,1)$ \\
$\begin{array}{l}\text { Tuvo heridas durante el trabajo } \\
\text { Manipulación de roedores en el campo de }\end{array}$ & 233 & $(13,5)$ \\
\begin{tabular}{l} 
cultivo \\
\hline
\end{tabular} & 178 & $(69,0)$ \\
\hline
\end{tabular}

$(11,7 \%, 27 / 230)$ y laceraciones en piel $(7,4 \%, 17 / 230)$. El 36,5\% (95/260) refirió almorzar en el campo de cultivo. El $69,5 \%$ refirió capturar ratas u otros roedores durante el trabajo. Los varones tuvieron mayor tiempo de permanencia en la actividad que las mujeres (25,29 \pm 13,16 años frente a $17,73 \pm 11,23$ años, $p=0,001$ ), jornadas de mayor duración $(7,32 \pm 1,99$ horas al día, frente a 6,32 $\pm 2,41$ horas/día, $p=0,02)$, menor uso de calzado en el trabajo $(62,0 \%$ comparado con $38,5 \%, p<0,01)$ y refirieron manipular ratas durante el trabajo con mayor frecuencia $(73,9 \%$ frente a $44,7 \%, p<0,01)$.

\section{CARACTERÍSTICAS CLÍNICAS}

Al momento de la evaluación, solo 57 participantes refirieron algún tipo de síntomas, 35 (13,5\%) de los cuales tuvieron algunos de los síntomas incluidos en los criterios de Faine modificado. Los síntomas más frecuentes fueron: cefalea $(11,2 \%, 29 / 260)$; dolor lumbar $(8,1 \%$, $21 / 260)$; dolor articular $(5,0 \%, 13 / 260)$; malestar general $(3,5 \%, 9 / 260)$; dolor en pantorrillas $(3,1 \%, 8 / 260)$; dolor abdominal $(2,3 \%, 6 / 260)$; anorexia $(0,8 \%, 2 / 260)$; astenia $(0,8 \%, 2 / 260)$, y dolor muscular $(0,8 \%, 2 / 260)$.

\section{SEROPREVALENCIA DE LEPTOSPIROSIS}

La seroprevalencia en esta población mediante la prueba MAT fue de $64,6 \%(168 / 260)$ con una variación entre 58,6 a $70,6 \%$ (IC 95\%). Con la prueba ELISA, se encontró 39 casos con anticuerpos IgM anti-Leptospira (15,0\%, IC 95\%: 10,5-19,5\%). El porcentaje de positivos a ELISA IgM fue significativamente mayor entre los que fueron reactivos a MAT, comparada con los que no lo fueron $(20,8 \%$ frente a $4,3 \%, p<0,01)$. No hubo diferencias 


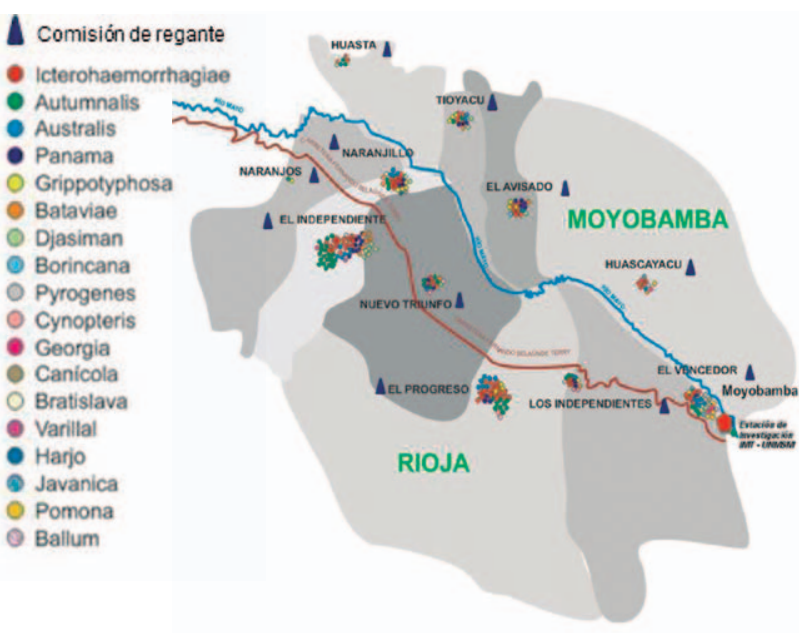

Figura 1. Distribución espacial de serovares de Leptospira en comisiones de regantes del valle del Alto Mayo, 2010.

significativas en la seroprevalencia de sintomáticos y no sintomáticos con MAT $(60,0 \%$ frente a $65,3 \%, p=0,54)$, ni con ELISA IgM (14,3\% frente a $15,1 \%, p=0,95)$.

Los casos de reactividad a MAT se presentaron en las once comisiones (Figura 1). En las comisiones de regantes de Moyobamba la seroprevalencia fue de $62,6 \%$ (62/99), mientras que en las de Rioja fue de 65,8\% (106/161). La reactividad se observó para 19 serovares distintos. En ambos distritos las comisiones tuvieron más de un serovar (Moyobamba: cuatro en El Independiente, tres en El Nuevo Triunfo, tres en El Progreso, cinco en Naranjillo y dos en Naranjos; Rioja: cinco en Los indepen- dientes, cinco en El Vencedor, cinco en El Avisado, tres en Tioyacu, tres en Huascayacu y dos en Huasta).

Los tipos de serovar más frecuentes fueron: Icterohaemorrhagiae, Autumnalis, Australis, Panama y Grippotyphosa (Tabla 3). De los 168 agricultores con MAT reactivo, $84(50,0 \%)$ fueron reactivos a un tipo de serovar, $58(34,5 \%)$ a dos tipos, $17(10,1 \%)$ a tres tipos, cuatro $(2,4 \%)$ a cuatro tipos y cinco $(3,0 \%)$ a cinco tipos. Los agricultores con mayor número de serovares tuvieron una edad media mayor y menor tiempo como agricultor dedicado al cultivo de arroz, aunque las diferencias no fueron significativa ( $p=0,23$ y 0,98 respectivamente).

\section{FACTORES ASOCIADOS}

El análisis bivariado (Tabla 4) mostró que tres factores relacionados con la actividad laboral de la población estudiada estuvieron estadísticamente asociados con la mayor seroprevalencia de leptospirosis: el tiempo de agricultor de arroz, el trabajar descalzo y el manipular ratas en el campo de cultivo. No se encontró asociación significativa con haber sufrido heridas durante el trabajo.

De los factores demográficos estudiados, estuvieron relacionados el sexo y el grado de instrucción; este último en el límite de significación estadística. El análisis estratificado de esta última relación, según sexo, mostró que la relación es significativa independientemente del sexo (OR ajustado con Mantel-Haenszel: 1,7, IC 95\%: 1,0-2,9). El promedio de edad de los reactivos a MAT fue similar a los no reactivos. Se observó mayor seroprevalencia en

Tabla 3. Frecuencia de serovares encontrados en 260 agricultores de arroz con prueba de microaglutinación reactiva a leptospirosis. Alto Mayo, San Martín, Perú 2010

\begin{tabular}{|c|c|c|c|c|c|c|}
\hline \multirow{2}{*}{ Serovar } & \multicolumn{4}{|c|}{ Dilución } & \multirow{2}{*}{$\begin{array}{c}\text { Número de } \\
\text { muestras reactivas }\end{array}$} & \multirow{2}{*}{ Prevalencia (IC 95\% } \\
\hline & $1 / 100$ & $1 / 200$ & $1 / 400$ & $\geq 1 / 800$ & & \\
\hline Icterohaemorrhagiae & 47 & 12 & 18 & 13 & 90 & $34,6(28,6-40,6)$ \\
\hline Autumnalis & 28 & 7 & 8 & 8 & 51 & $19,6(14,6-24,6)$ \\
\hline Panama & 26 & 3 & 3 & 1 & 33 & $12,7(8,5-16,9)$ \\
\hline Australis & 28 & 4 & 0 & 0 & 32 & $12,3(8,1-16,5)$ \\
\hline Grippotyphosa & 16 & 1 & 1 & 2 & 20 & $7,7(4,3-11,1)$ \\
\hline Bataviae & 9 & 1 & 2 & 2 & 14 & $5,4(2,5-8,3)$ \\
\hline Djasiman & 9 & 1 & 2 & 1 & 13 & $5,0(2,2-7,8)$ \\
\hline Pyrogenes & 7 & 0 & 1 & 2 & 10 & $3,8(1,3-6,4)$ \\
\hline Cynopteri & 5 & 0 & 1 & 0 & 6 & $2,3(0,3-4,3)$ \\
\hline Pomona & 5 & 0 & 0 & 0 & 5 & $1,9(0,6-4,4)$ \\
\hline Georgia & 4 & 0 & 0 & 0 & 4 & $1,5(0,4-3,9)$ \\
\hline Canicola & 2 & 0 & 0 & 1 & 3 & $1,2(0,2-3,3)$ \\
\hline Borincana & 2 & 0 & 0 & 0 & 2 & $0,8(0,1-2,8)$ \\
\hline Javanica & 2 & 0 & 0 & 0 & 2 & $0,8(0,1-2,8)$ \\
\hline Wolfii & 2 & 0 & 0 & 0 & 2 & $0,8(0,1-2,8)$ \\
\hline Ballum & 1 & 0 & 0 & 0 & 1 & $0,4(0,01-2,1)$ \\
\hline Bratislava & 0 & 1 & 0 & 0 & 1 & $0,4(0,01-2,1)$ \\
\hline Harjo & 0 & 1 & 0 & 0 & 1 & $0,4(0,01-2,1)$ \\
\hline Varillal & 0 & 0 & 0 & 1 & 1 & $0,4(0,01-2,1)$ \\
\hline
\end{tabular}


Tabla 4. Análisis bivariado de características asociadas a la seroprevalencia de leptospirosis (reactividad a prueba de microaglutinación), en agricultores de arroz del valle de Alto Mayo, 2010

\begin{tabular}{|c|c|c|c|}
\hline Factores estudiados & $\mathbf{N}$ & $\begin{array}{l}\text { Reactivos a } \\
\text { MAT (\%) }\end{array}$ & $\begin{array}{l}\text { OR crudo } \\
\text { (IC } 95 \%)\end{array}$ \\
\hline \multicolumn{4}{|l|}{ Edad en años } \\
\hline$\leq 39$ & 78 & $48(61,5)$ & 1,00 \\
\hline $40-59$ & 126 & $83(65,9)$ & $1,07(0,86-1,32)$ \\
\hline$\geq 60$ & 56 & $37(66,1)$ & $1,07(0,83-1,38)$ \\
\hline Sexo masculino & 221 & $152(68,8)$ & $3,17(1,57-6,36)$ \\
\hline $\begin{array}{l}\text { Grado de instrucción } \\
\text { primaria o analfabeto }\end{array}$ & 158 & $109(69,0)$ & $1,68(0,99-2,82)$ \\
\hline \multicolumn{4}{|l|}{ Años de residencia } \\
\hline$\leq 19$ & 73 & $45(61,6)$ & 1,00 \\
\hline $20-29$ & 80 & $54(67,5)$ & $1,09(0,86-1,39)$ \\
\hline$\geq 30$ & 107 & $69(64,5)$ & $1,04(0,83-1,32)$ \\
\hline \multicolumn{4}{|l|}{$\begin{array}{l}\text { Nivel de protección del } \\
\text { hogar }\end{array}$} \\
\hline Alto & 51 & $31(60,8)$ & 1,00 \\
\hline Medio & 100 & $68(68,0)$ & $1,11(0,86-1,45)$ \\
\hline Bajo & 109 & $69(63,3)$ & $1,04(0,80-1,35)$ \\
\hline $\begin{array}{l}\text { Ha observado ratas } \\
\text { dentro del hogar }\end{array}$ & 245 & $157(64,1)$ & $0,65(0,20-2,09)$ \\
\hline $\begin{array}{l}\text { Hogar próximo a } \\
\text { desagües, } \\
\text { alcantarillas o } \\
\text { basurales }\end{array}$ & 118 & $80(67,8)$ & $1,29(0,77-2,16)$ \\
\hline $\begin{array}{l}\text { Almacena comida } \\
\text { alrededor de la casa }\end{array}$ & 69 & $50(72,5)$ & $1,63(0,89-2,98)$ \\
\hline \multicolumn{4}{|l|}{ Años como agricultor } \\
\hline$<15$ & 59 & $30(50,8)$ & 1,00 \\
\hline $15-29$ & 102 & $74(72,5)$ & $1,43(1,08-1,88)$ \\
\hline 30 a más & 97 & $64(66,0)$ & $1,29(0,97-1,73)$ \\
\hline \multicolumn{4}{|l|}{$\begin{array}{l}\text { Tipo de calzado durante } \\
\text { trabajo }\end{array}$} \\
\hline $\begin{array}{l}\text { Zapato, zapatilla o } \\
\text { sandalia }\end{array}$ & 35 & $14(40,0)$ & 1,00 \\
\hline Bota & 73 & $44(60,3)$ & $1,51(0,96-2,35)$ \\
\hline Descalzo & 152 & $110(72,4)$ & $1,81(1,19-2,75)$ \\
\hline $\begin{array}{l}\text { Tuvo heridas durante } \\
\text { el trabajo }\end{array}$ & 233 & $153(65,7)$ & $1,40(0,62-3,19)$ \\
\hline $\begin{array}{l}\text { Manipulación de } \\
\text { roedores en el campo } \\
\text { de cultivo }\end{array}$ & 178 & $125(70,2)$ & $2,24(1,29-3,88)$ \\
\hline
\end{tabular}

los no naturales de San Martín $(66,4 \%)$ respecto a los naturales $(56,3 \%)$, pero la diferencia no fue significativa. Ninguna de las condiciones del hogar (nivel de protección del hogar, observación de ratas dentro del hogar, proximidad a desagües, alcantarillas o basurales, y almacenamiento de comida alrededor de la casa) tuvo asociación significativa con la seroprevalencia.

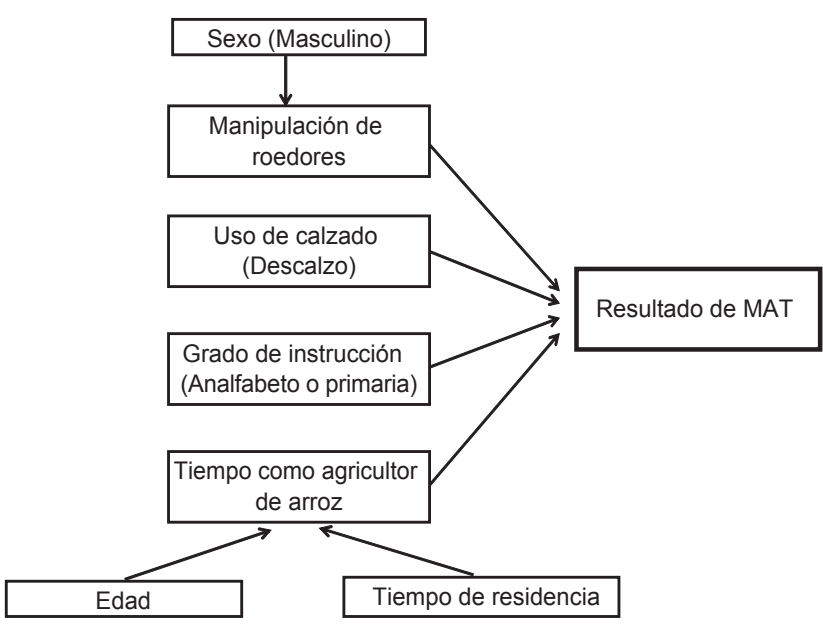

Figura 2. Modelo propuesto de factores asociados al resultado del MAT en agricultores de arroz de Alto Mayo, 2010

Teniendo en cuenta la red de relaciones observadas en este estudio (Figura 2), se construyó un modelo multivariado, descrito en metodología, y cuyo resumen puede verse en la Tabla 5. El OR ajustado (ORa) en este modelo, muestra que el manipular ratas en el campo y el trabajar descalzo están significativamente asociados a la seroprevalencia de leptospirosis, independientemente del sexo, el tiempo como agricultor y del grado de instrucción. En el caso del trabajar descalzo, la fuerza de asociación fue mayor que en el análisis bivariado $(7,9$ frente a 1,8$)$.

El modelo de regresión logística incluye: grado de instrucción, tiempo como agricultor de arroz, manipulación de roedores y tipo de calzado.

Tabla 5. Análisis multivariado de factores asociados a la seroprevalencia de leptospirosis en agricultores de arroz del valle de Alto Mayo, 2010

\begin{tabular}{lc}
\hline Variable & OR ajustado (IC 95\%) \\
\hline Sexo & 1,00 \\
Femenino & $4,46(1,30-15,34)$ \\
Masculino & 1,00 \\
Grado de instrucción & $1,81(0,98-3,34)$ \\
Secundaria o superior & 1,00 \\
\hline Analfabeto o primaria & $2,01(0,95-4,24)$ \\
Años como agricultor & $1,16(0,54-2,51)$ \\
$<15$ & 1,00 \\
15 a 29 & $7,89(1,64-37,95)$ \\
$\geq 30$ & 1,00 \\
Manipulación de roedores & $1,91(0,76-4,81)$ \\
No & $2,86(1,20-6,82)$ \\
Sí & $0,17(0,03-0,93)$ \\
\hline Tipo de calzado en trabajo & \\
Zapato, zapatilla, sandalia & \\
Bota & \\
Descalzo & \\
Interacción entre sexo y \\
manipulación de roedores
\end{tabular}




\section{DISCUSIÓN}

La elevada seroprevalencia de leptospirosis observada en la población de trabajadores de arroz del valle del Alto Mayo $(64,6 \%)$, indica una condición hiperendémica, mayor a la encontrada en estudios realizados en poblaciones similares en el país. Así, en el estudio realizado en arroceros de Lambayeque el año 1986 la prevalencia fue $56,0 \%{ }^{(24)}$, y en el estudio realizado en la región San Martín en el año $2000{ }^{(22)}$, en el que también se incluyó localidades del valle del Alto Mayo, la prevalencia fue $25,2 \%$. La diferencia con este último estudio podría deberse a que para detectar los anticuerpos usaron ELISA IgG de menor sensibilidad que MAT; también, a que la estimación de la seroprevalencia fue para cuatro provincias de la región San Martín y que la unidad de muestreo fue la vivienda y no el agricultor. Sin embargo, es probable que la incidencia de infección por Leptospira se haya incrementado en la región en los últimos 10 años, debido a la intensificación del cultivo de arroz y a la mayor migración de población en edad productiva, procedente de zonas de baja endemicidad.

Además de la elevada prevalencia, destaca en este estudio la cantidad de serovares hallados. Los serovares más comunes fueron: Icterohaemorrhagiae $(34,6 \%)$, Autumnalis (19,6\%), Australis (12,7\%), Panama (12,3\%) y Grippotyphosa (7,7\%). Un estudio previo en la región San Martín encontró los mismos serovares y otros dos adicionales (Tarassovi, Andama); sin embargo, en dicho estudio los serovares más frecuentes fueron Bataviae, Bratislava, Georgia y Djasiman, mientras que el Icterohaemorrhagiae se encontró solo en dos participantes ${ }^{(22)}$. A pesar que la presencia de serovares es similar en varias regiones endémicas del Perú, su prevalencia varía, por ejemplo, en Madre de Dios ${ }^{(19)}$ y Ucayali (21) los serovares más frecuentes son Georgia y Bratislava, mientras en la selva alta de Ayacucho son los serovares Icterohaemorrhagiae, Djasiman, Varillal y Panama ${ }^{(20)}$. Estudios en zonas endémicas realizados en población general asintomática y en grupos expuestos muestran que en lugares con prevalencias elevadas $(>30 \%)$ circulan varios tipos de serovares, desde 13 tipos en la región Madre de Dios hasta 19 serovares en San Martín (19-21).

La circulación de varios serovares, aunada a que el $15 \%$ de agricultores presentaron anticuerpos IgM, que son producidos en etapas tempranas de infección (3-10 días de infección) ${ }^{(14)}$, indican que la transmisión de esta infección es activa en esta región. También supone la presencia de una cantidad importante de reservorios que contaminan las fuentes de agua y en particular el agua que se usa en el cultivo de arroz.
Un reto adicional para el control de esta infección radica en que $87,5 \%$ de agricultores serorreactivos no tenía síntomas o tuvieron síntomas leves o inespecíficos, que podrían deberse a manifestaciones propias del trabajo físico intenso realizado. Ninguno de los participantes presentó sintomatología febril, observación también descrita en otras áreas endémicas ${ }^{(10,29,30)}$. Este hecho puede estar relacionado a que solo el $10 \%$ de reacciones positivas a MAT tuvieron títulos $\geq 1 / 800$ (indicador de infección reciente); la mayoría presentó concentraciones bajas de anticuerpos. También es importante considerar que la inmunidad frente a la leptospirosis es de tipo humoral y serovar específica, pudiendo brindar inmunidad cruzada para serovares relacionados ${ }^{(12,31,32)}$, especialmente en este estudio donde se encontraron hasta 19 serovares diferentes y el $50 \%$ de agricultores reactivos a más de un serovar.

Otro hallazgo relevante es la fuerte asociación observada entre la seroprevalencia y dos prácticas laborales: el trabajar descalzo y la manipulación de roedores en el campo de cultivo. Estas prácticas, según el análisis multivariado, resultaron asociadas independientemente del sexo, el tiempo como agricultor de arroz y el grado de instrucción. No hay estudios previos en el que se haya precisado el riesgo que implican estas prácticas, aunque han sido señaladas en diversas revisiones generales y estudios realizados en otros países ${ }^{(11,20,33,34)}$. La importancia de esta observación radica en que se trata de factores modificables a través de intervenciones preventivas en esta población, que incluyan la implementación de medios de protección, como el uso de calzado, y la capacitación para evitar la manipulación de roedores en los campos de cultivo. El impacto de estas medidas podría ser considerable, si se toma en cuenta que $58 \%$ de los agricultores trabaja descalzo y casi $70 \%$ refiere manipular roedores en los campos de cultivo.

Adicionalmente, este estudio mostró que si bien la mayor seroprevalencia se presentó en quienes no usan calzado, también fue alta entre quienes usan botas, aunque la asociación no fue significativa (ORa: 1,91. IC95\%: 0,764,81). Es importante evaluar este tipo de calzado, pues si bien ofrecen mayor protección, su mal estado o su uso inadecuado podrían tener un efecto contraproducente en el caso de los cultivadores de arroz, quienes mantienen sus miembros inferiores expuestos al agua contaminada con orina de reservorios durante más de $6 \mathrm{~h} /$ día. También llama la atención en este estudio, la falta de asociación con haber sufrido heridas durante el trabajo, lo cual podría deberse a que más del $90 \%$ refiere este tipo de accidente; pero no se debe descartar la posibilidad que el mantener los pies sumergidos en el agua contaminada por tiempo prolongado produzca lesiones que faciliten el ingreso de la Leptospira con mayor eficacia que las lesiones por accidentes. 
El tiempo de trabajo como agricultor de arroz estuvo asociado con la mayor seroprevalencia en el análisis bivariado, pero dejó de estarlo luego del análisis multivariado, donde otros factores cobraron mayor importancia. Está descrito, en estudios de leptospirosis en grupos expuestos al riesgo de infección, que independientemente del tiempo de exposición ${ }^{(30)}$ son importantes la susceptibilidad y el contacto con la fuente de infección, aun si esta sucede una única vez. Esto ocurre, por ejemplo, en personas que practican el ecoturismo o en aquellas que sufren las consecuencias de desastres naturales ${ }^{(33,35,36)}$.

Los hombres tuvieron mayor seroprevalencia que las mujeres, tanto en el análisis bivariado como el multivariado. Este resultado puede explicarse porque la gran mayoría de agricultores incluidos en el estudio fue de sexo masculino (85\%), y también porque muchos factores asociados estuvieron relacionados conel sexo, aunque luego del análisis estratificado se descartó que esta variable sea confusora y más bien se identificó que tenía un efecto modificador en la relación con la práctica de trabajar descalzo.

Finalmente, hay aspectos que el estudio no ha podido establecer tanto por el diseño como por el tamaño de la muestra. Por ser un estudio transversal no es posible afirmar que las asociaciones observadas sean causales; sin embargo, hay que considerar que se trata de factores identificados en otros estudios y consistentes con el modelo biológico de transmisión establecido por estudios previos, de modo que es importante tomarlos en cuenta para establecer intervenciones preventivas. Otro aspecto se relaciona con el método inmunológico y la forma de aplicación empleados en este estudio. Como ya se ha señalado, la medición en una sola oportunidad de anticuerpos mediante el uso de MAT y/o ELISA IgM en una zona endémica dificulta la determinación de casos de leptospirosis aguda y la identificación del tipo de serovar responsable de la actual infección, que en muchos casos fue asintomática ${ }^{(14)}$. También hay que señalar que el estudio no exploró el estado nutricional de los agricultores, ni calculó la carga de leptospiras a la que están expuestos en forma regular durante sus labores; tampoco incluyó el estudio de anticuerpos en reservorios que confirmen la presencia de los serovares circulantes identificados en humanos.
En conclusión, en el presente estudio se evidencia que esta región es un área hiperendémica de leptospirosis con elevada prevalencia de cuadros asintomáticos, donde la exposición ambiental a esta bacteria es frecuente. El hallazgo de una diversa cantidad de serovares circulantes, de múltiples serovares en un solo individuo (reinfecciones) y la posibilidad de protección serovar cruzada explicarían la elevada prevalencia de infección asintomática, a través de mecanismos de inmunidad protectora adquirida. Los factores asociados a la seroprevalencia más importantes fueron la práctica de trabajar descalzo y la de manipular roedores en los campos de cultivo. En consecuencia, se recomienda implementar intervenciones que incidan en estos dos factores a fin de reducir el riesgo de infección en los cultivadores de arroz. Este estudio debe complementarse con la investigación de reservorios, de la carga de enfermedad en febriles y del papel de la infección asintomática en la epidemiologia de la leptospirosis en zonas endémicas.

Agradecimientos: los autores agradecen al Proyecto Especial Alto Mayo (PEAM), a la Junta de Usuarios de la Cuenca del Alto Mayo (JUCAM), y al Gobierno Regional de San Martín, quienes prestaron su ayuda y dieron las facilidades para la realización del presente estudio. Asimismo, a los médicos Luis Mendoza, Ricardo Flores y Augusto Pillaca, del Hospital I de Moyobamba; al Lic. Luis Marín, al técnico Miguel Farfán del INS, y a la Mg. María Esther Salazar, del IMT UNMSM, por su profesional apoyo en el trabajo de campo.

Contribuciones de autoría: JOAV, FRRR, PWC y RTC participaron en la concepción del estudio. FRR, PWC y RTC contribuyeron en la recolección de datos. MCZ participó en el procesamiento y análisis de laboratorio. JOAV y FRRR participaron en el análisis, interpretación de datos y redacción del artículo. JOAV, FRRR, PWC y RTC revisaron en forma crítica versiones preliminares del manuscrito. Todos los autores revisaron en forma crítica versiones preliminares del manuscrito y aprobaron su versión final.

Fuentes de financiamiento: el Instituto Nacional de Salud (INS) a través del Centro Nacional de Salud Pública asumió los gastos de recolección y procesamiento de muestras. El Instituto Peruano de Investigación en Ciencias Médicas (IPICMED) y el Gobierno Regional de San Martín asumieron los gastos operativos del trabajo de campo y recolección de muestras.

Conflictos de interés: los autores declaran no tener conflictos de interés.

\section{REFERENCIAS BIBLIOGRÁFICAS}

1. Picardeau M. Diagnosis and epidemiology of leptospirosis. Med Mal Infect. 2013 Jan;43(1):1-9. doi: 10.1016/j. medmal.2012.11.005.

2. Musso D, La Scola B. Laboratory diagnosis of leptospirosis: a challenge. J Microbiol Immunol Infect. 2013 Aug;46(4):245-52. doi: 10.1016/j. jmii.2013.03.001.
3. Bharti AR, Nally JE, Ricaldi JN, Matthias MA, Diaz MM, Lovett MM, et al. Leptospirosis: a zoonotic disease of global importance. Lancet Infect Dis. 2003 Dec;3(12):757-71.

4. Pappas G, Papadimitriou P, Siozopoulou V, Christou L, Aktritidis N. The globalization of leptospirosis: woldwide incidence trens. Int J Infect Dis. $2008 \mathrm{Jul} ;(12): 351-7$.
5. Guerra MA. Leptospirosis: public health perspectives. Biologicals. 2013 Sep;41(5):295-7. doi: 10.1016/j.biologicals.2013.06.010.

6. Costa F, Martinez-Silveira MS, Hagan JE, Hartskeerl RA, Dos Reis MG, Ko AI. Surveillance for leptospirosis in the Americas, 1996-2005: a review of data from ministries of health. Rev Panam Salud Publica. 2012 Sep;32(3):169-77. 
7. Abela-Ridder B, Sikkema R, Hartskeerl RA. Estimating the burden of human leptospirosis. Int J Antimicrob Agents. 2010 Nov;36 Suppl 1:S5-7. doi: 10.1016/j.ijantimicag.2010.06.012.

8. Lacerda HG, Monteiro GR, Oliveira CC, Suassuna FB, Queiroz JW, Barbosa JD, et al. Leptospirosis in a subsistence farming community in Brazil. Trans R Soc Trop Med Hyg. 2008 Dec;102(12):1233-8. doi: 10.1016/j. trstmh.2008.05.010.

9. Victoriano AF, Smythe LD, Gloriani-Barzaga N, Cavinta LL, Kasai T, Limpakarnjanarat $\mathrm{K}$, et al. Leptospirosis in the Asia Pacific region. BMC Infect Dis. 2009 Sep 4;9:147. doi: 10.1186/1471-2334-9-147.

10. Kawaguchi L, Sengkeopraseuth, B, Tsuyuoka, R, Koizumi N, Akashi H, Vongphrachanh $\mathrm{P}$, et al. Seroprevalence of leptospirosis and risk factor analysis in flood-prone rural areas in Lao PDR. Am J Trop Med Hyg. 2008 Jun;78(6):957-61.

11. Hartskeerl RA, Collares-Pereira M, Ellis WA. Emergence, control and reemerging leptospirosis: dynamics of infection in the changing world. Clin Microbiol Infect. 2011 Apr;17(4):494501. doi: 10.1111/j.14690691.2011.03474.x.

12. Ganoza CA, Matthias MA, Saito M, Cespedes M, Gotuzzo E, Vinetz JM. Asymptomatic renal colonization of numans in the peruvian Amazon by Leptospira. PLoS Negl Trop Dis. 2010 Feb 23;4(2):e612. doi: 10.1371/journal.pntd.0000612.

13. Libraty DH, Myint KS, Murray CK, Gibbons RV, Mammen MP, Endy TP, et al. A comparative study of leptospirosis and Dengue in thai children. PLoS Negl Trop Dis. 2007 Dec 26;1(3):e111.

14. Ashford DA, Kaiser RM, Spiegel RA, Perkins BA, Weyant RS, Bragg SL, et al. Asymptomatic infection and risk factors for leptospirosis in Nicaragua. Am J Trop Med Hyg. 2000 NovDec;63(5-6):249-54.

15. Silva HR, Tavares-Neto J, Bina JC, Meyer R. Leptospirose-infecccão e forma subclínica em crianccas de Salvador, Bahia. Rev Soc Bras Med Trop. 2003 Mar-Apr;36(2):227-33.

16. Budihal SV, Perwez K. Leptospirosis diagnosis: competancy of various laboratory tests. J Clin Diagn Res. 2014 Jan;8(1):199-202. doi: 10.7860/ JCDR/2014/6593.3950.

17. Perú, Ministerio de Salud, Dirección General de Epidemiología. Resumen de las enfermedades o eventos sujetos a notificación obligatoria. Bol Epidemiol (Lima). 2013;22(50):1044-8.

18. Perú, Ministerio de Salud, Dirección General de Epidemiología. Enfermedades sujetas a vigilancia epidemiológica. Bol Epidemiol (Lima). 2013;22(52):1098-1102

19. Céspedes M, Ormaeche M, Condori P, Balda L, Glenny M. Prevalencia de leptospirosis y factores de riesgo en personas con antecedentes de fiebre en la provincia de Manu, Madre de Dios, Perú. Rev Peru Med Exp Salud Publica. 2003;20(4):180-5.

20. Vargas-Cuba F, García-Apaico V, Céspedes M, Palomino-Enciso M, AyalaHuaytalla T. Seroprevalencia y factores asociados con leptospirosis en pacientes con síndrome febril en Ayacucho, Perú 2005. Rev Peru Med Exp Salud Publica. $2008 ; 25(2): 190-4$.

21. Céspedes M, Fernández R, Rimarachín R, Taipe H, Cenepo J, Mori y Gonzales M, et al. Leptospirosis: Una enfermedad zoonótica hiperendémica en la provincia de Coronel Portillo. Ucayali, Perú. Rev Peru Med Exp Salud Publica. 2004;21(2):62-70.

22. Cruz R, Fernández F, Arévalo H. Hiperendemicidad de leptospirosis y factores de riesgo asociados en localidades arroceras del departamento de San Martín-Perú. Rev Peru Med Exp Salud Publica. 2002;19(1):10-6.

23. Perú, Ministerio de Agricultura. Arroz en el Perú. Lima: Ministerio de Agricultura; 2010.

24. Fernández G. Prevalencia de anticuerpos leptospirales en agricultores de la provincia de Ferreñafe 1985. Tesis para obtener el título de Licenciado en Biología. Lambayeque; Perú: Universidad Nacional Pedro Ruiz Gallo; 1986;

25. Céspedes M, Glenny M, Felices V, Balda L, Suárez V. Prueba de ELISA indirecta para la detección de anticuerpos IgM para el diagnóstico de leptospirosis humana. Rev Peru Med Exp Salud Publica. 2002;19(1):23-7.

26. Céspedes Zambrano M. ELISA IgM para diagnóstico de Leptospira. Método de ensayo. Lima: Instituto Nacional de Salud; 2008.

27. Cole J, Sulzer C, Pursell A. Improved microtechnique for the leptospiral microscopic agglutination test. Appl Microbiol. 1973;25(6):976-80.

28. Sethi S, Sharma N, Kakkar N, Taneja J, Chatterjee SS, Banga SS, et al. Increasing trends of leptospirosis in Nor- thern India: a clinico-epidemiological study. PLoS Negl Trop Dis. 2010 Jan 12;4(1):e579. doi: 10.1371/journal. pntd.0000579.

29. Spichler A, Athanazio DA, Vilaca P, Seguro A, Vinetz J, Leake JA. Comparative analysis of severe pediatric and adult leptospirosis in Sao Paulo, Brazil. Am J Trop Med Hyg. 2012 Feb;86(2):306-8. doi: 10.4269/ajtmh.2012.11-0308.

30. Sharma S, Vijayachari P, Sugunan AP, Natarajaseenivasan K, Sehgal SC. Seroprevalence of leptospirosis among high-risk population of Andaman Islands, India. Am J Trop Med Hyg. 2006 Feb;74(2):278-83.

31. Koizumi N, Watanabe H. Leptospirosis vaccines: past, present and future. J Postgrad Med. 2005 JulSep;51(3):210-4.

32. Goris MG, Wagenaar JF, Hartskeerl RA, van Gord EC, Schuller S, Monahan A. et al. Potent innate immune response to pathogenic leptospira in human whole blood. PLoS One. 2011 Mar 31;6(3):e18279. doi: 10.1371/ journal.pone.0018279.

33. Lau CL, Smythe LD, Craig SB, Weinstein P. Climate change, flooding, urbanisation and leptospirosis: fuelling the fire? Trans R Soc Trop Med Hyg. 2010 Oct; $104(10): 631-8$. doi: 10.1016/j. trstmh.2010.07.002.

34. Bovet P, Yersin C, Merien F, Davis CE, Perolat P. Factors associated with clinical leptospirosis: a population-based case-control study in the Seychelles (Indian Ocean). Int J Epidemiol. 1999 Jun;28(3):583-90.

35. Narita M, Fujitani S, Haake DA, Paterson DL. Leptospirosis after recreational exposure to water in the Yaeyama Islands, Japan. Am J Trop Med Hyg. 2005 Oct;73(4):652-6.

36. Cascio A, Bosilkovski M, RodriguezMorales AJ, Pappas G. The socioecology of zoonotic infections. Clin Microbiol Infect. 2011 Mar;17(3):33642. doi: $10.1111 / \mathrm{j} .1469$ 0691.2010.03451.x.

Correspondencia: Jorge Alarcón-Villaverde Dirección: Instituto de Medicina Tropical "Daniel A. Carrión"-Universidad Nacional Mayor de San Marcos. Jr. José Santos Chocano 199. 4to. piso. Urb. San Joaquin - Bellavista Callao 2, Perú.

Correoelectrónico:jalarconv@unmsm.edu.pe 